Société d'histoire de la révolution de 1848 et des révolutions du XIXe siècle

\title{
Nicole St-Onge, Carolyn Podruchny et Brenda Macdougall (eds), Contours of a People. Metis Family, Mobility and History
}

Norman (Okla.), University of Oklahoma Press, 2012, 482 p. ISBN

978-0-8061-4279-1. 39,95 dollars

Tangi Villerbu

\section{OpenEdition}

Journals

Édition électronique

URL : http://journals.openedition.org/rh19/4511

DOI : 10.4000/rh19.4511

ISSN : $1777-5329$

Éditeur

La Société de 1848

Édition imprimée

Date de publication : 1 juin 2013

Pagination : 230-232

ISSN : 1265-1354

\section{Référence électronique}

Tangi Villerbu, « Nicole St-Onge, Carolyn Podruchny et Brenda Macdougall (eds), Contours of a People. Metis Family, Mobility and History ", Revue d'histoire du XIXe siècle [En ligne], 46 | 2013, mis en ligne le 13 janvier 2014, consulté le 22 septembre 2020. URL : http://journals.openedition.org/rh19/4511 ; DOI : https://doi.org/10.4000/rh19.4511

Ce document a été généré automatiquement le 22 septembre 2020.

Tous droits réservés 


\title{
Nicole St-Onge, Carolyn Podruchny et Brenda Macdougall (eds), Contours of a People. Metis Family, Mobility and \\ History
}

\author{
Norman (Okla.), University of Oklahoma Press, 2012, 482 p. ISBN
}

978-0-8061-4279-1. 39,95 dollars

\section{Tangi Villerbu}

\section{RÉFÉRENCE}

Nicole St-Onge, Carolyn Podruchny et Brenda Macdougall (eds), Contours of a People.

Metis Family, Mobility and History, Norman (Okla.), University of Oklahoma Press, 2012, 482 p. ISBN 978-0-8061-4279-1. 39,95 dollars

1 Les Métis forment au Canada un groupe national distinct, reconnu comme tel au sein de l'ensemble aborigène depuis 1982. Leur mémoire est vivace ; leur histoire, longtemps, le fut beaucoup moins: Marcel Giraud avait en 1945 montré la voie dans un travail de grande ampleur aujourd'hui dépassé ${ }^{1}$ mais il fallut attendre la fin des années 1970 pour qu'un renouveau se fasse sentir, avec les travaux de Jacqueline Peterson, Jennifer Brown ou Sylvia Van Kirk, à la rencontre du métissage massif qui se produisit dans le cadre du commerce de la fourrure sur les terres de la Compagnie de la Baie d'Hudson (et avant 1822 de celle du Nord-Ouest) et donna naissance aux Métis ${ }^{2}$ - de manière non systématique car tout métissage ne crée pas de nation distincte. Ces dernières années, d'importants travaux ont de nouveau vu le jour, autour notamment des réseaux familiaux et de la mobilité du groupe ${ }^{3}$, et cet ouvrage collectif vient à point nommé marquer une étape importante du champ historiographique métis.

Il serait illusoire de vouloir analyser ici chacune des quinze contributions (en comptant l'introduction) de cet ouvrage majeur. Reste que des tendances se dégagent. Une des 
plus importantes est la prise en compte de l'ensemble du continent dans la réflexion sur cette histoire de métissage : les travaux offrent un vaste panorama qui promène le lecteur aux États-Unis (Wisconsin, Montana), au Canada bien sûr (dans les Plaines, mais aussi dans le Nord-Ouest et, plus rare et très innovant, en Colombie-Britannique) mais aussi dans l'Amérique russe (devenue Alaska). Certes, des champs ne sont pas encore couverts - on pense par exemple à l'Oregon, pourtant travaillé par Melinda Marie Jetté ${ }^{4}$, ou au Minnesota - mais la variété des situations permet d'envisager pleinement l'ethnogenèse métisse en son contexte et de mettre en valeur les acquis récents comme les questions toujours sans réponse.

3 Il est évidemment impossible de dater précisément l'acte de naissance d'un peuple. Cela est d'autant plus difficile dans le cas des Métis. Les rejetons issus de couples mixtes hommes euro-américains (surtout Canadiens) et femmes indiennes - sont présents dans l'Ouest depuis le xvIII ${ }^{e}$ siècle, mais seule une partie d'entre eux pratique l'endogamie et forme un groupe qui se définit comme Métis à partir du début du XIX siècle. La bataille de Seven Oaks en 1816, qui oppose des métis (sans majuscule, l'ethnogenèse n'a pas eu lieu) à la Compagnie de la Baie d'Hudson, et la Chanson de la Grenouillère qui la met en vers immédiatement après, et est étudiée ici par Gerhard Ens et par Étienne Rivard, ne peuvent faire office que de marqueurs symboliques d'un processus de longue durée. La géographie de ce processus est fondamentale : en effet pourquoi les mêmes conditions - des couples mixtes, des individus métissés qui œuvrent dans le commerce de la fourrure et se marient souvent entre eux - ne créentelles pas toujours les mêmes effets? Lucy Elderveld Murphy, en reprenant le dossier des familles des Prairie du Chien (Wisconsin) qu'elle connaît si bien ${ }^{5}$, montre que les contraintes sociales et juridiques imposées par les États-Unis ont très tôt coupé court à l'ethnogenèse du côté sud de la frontière, alors même que les familles étaient identiques de part et d'autre du $49^{\mathrm{e}}$ parallèle : certains membres sont devenus Métis au nord, d'autres au sud soit indiens soit half-breeds, et dans ce dernier cas amenés à disparaître sans créer de nouvelle identité nationale. Jacqueline Peterson souligne pour sa part à quel point il serait important de scruter ces espaces de transition entre lieux de l'ethnogenèse et lieux de la disparition.

Cette insistance sur des conceptions raciales rigides et transposées dans le domaine juridique et politique aux États-Unis est d'autant plus fascinante que l'ensemble des auteurs de Contours of a People met l'accent, in fine, sur la distance qu'il faut prendre absolument avec le critère «racial» dans la définition des Métis. Le métissage biologique ne peut être un facteur pertinent en la matière : il est autant présent chez ceux qui se disent Indiens ou Blancs que chez les Métis. L'identité repose donc sur d'autres facteurs qui se cristallisent tout le long du XIXe siècle. Brenda Macdougall insiste ainsi très fortement sur « un sens de soi formé par une connexion maternelle à la terre » (p. 440), la centralité donc des liens familiaux, autour en particulier de la figure maternelle ancestrale, comme vecteur d'enracinement d'une part et de sens géographique d'autre part. Car c'est une caractéristique majeure des Métis que leur sens de l'espace. Leur très vaste pays recouvre un grand Ouest qui constitue comme un tissu relationnel, un réseau parental spatialisé et qui ignore donc a priori les frontières géopolitiques que les États lui surimposent. Nicole St-Onge et Carolyn Podruchny ajoutent à cela le rapport au commerce des fourrures, qui fait naître les Métis, leur construit des réseaux de longue durée, les lie au capital marchand et leur permet une continuité de pratiques culturelles spécifiques (p. 80-81). La richesse de l'ouvrage est 
aussi de montrer que ces pratiques, comme la question de l'identité métisse, demeurent aujourd'hui en débat. La distinction entre « Métis » - membre de la nation - et « métis » - l'individu biologiquement métissé - réglée depuis maintenant près de trente ans, il reste que le nom même du peuple en question n'est pas fixé : « Métis » pour rappeler les origines majoritairement françaises du groupe, ou "Metis ", comme le préfèrent certains dont ici Brenda Macdougall, pour être plus inclusif mais en négligeant un héritage important?

\section{NOTES}

1. Marcel Giraud, Le métis canadien. Son rôle dans l'histoire des provinces de l'Ouest, Paris, Institut d'ethnologie, 1945.

2. Jacqueline Peterson, 'Prelude to Red River: a social portrait of the Great Lakes Métis', Ethnohistory, 25/1, hiver 1978, p. 41-67; Jennifer S.H. Brown, Strangers in blood. Fur Trade Company Families in Indian Country, Vancouver, University of British Columbia Press, 1980; Sylvia Van Kirk, 'Many Tender Ties': Women in Fur-Trade Society, 1670-1870, Winnipeg, Watson and Dwyer, 1980. Un ouvrage collectif venait dresser un premier bilan : Jacqueline Peterson et Jennifer S.H. Brown (eds), The New Peoples. Being and Becoming Métis in North America, Lincoln (Neb.), University of Nebraska Press, 1985.

3. Nicole St-Onge, Saint-Laurent, Manitoba. Evolving Métis Identities, 1850-1914, Regina, Canadian Plains Research Center, 2004; Heather Devine, The People Who Own Themselves. Aboriginal Ethnogenesis in a Canadian Family, 1660-1900, Calgary, University of Calgary Press, 2004; Brenda Macdougall, One of the Family. Metis culture in Nineteenth-Century Northwestern Saskatchewan, Vancouver, University of British Columbia Press, 2010; Brenda Macdougall et Nicole St-Onge, 'Rooted in mobility: Metis Buffalo-Hunting Brigades', Manitoba History, $\mathrm{n}^{\circ} 71$, hiver 2013, p. 21-32.

4. Melinda Marie Jetté, 'At the Hearth of the Crossed Races': A French-Indian Community in Nineteenth-Century Oregon, Corvallis (Or.), Oregon State University Press, à paraître.

5. Lucy Eldersveld Murphy, A Gathering of Rivers: Indians, Métis, and Mining in the Western Great Lakes, 1737-1832, Lincoln (Neb.), University of Nebraska Press, 2000. 